ETH-TH/99-06

February 1999

\title{
Higgs production with large transverse momentum in hadronic collisions at next-to-leading order *
}

\author{
D. de Florian, M. Grazzini and Z. Kunszt \\ Institute of Theoretical Physics, ETH, CH-8093 Zürich, Switzerland
}

\begin{abstract}
Inclusive associated production of a light Higgs boson $\left(m_{\mathrm{H}} \leq m_{t}\right)$ with one jet in $p p$ collisions is studied in next-to-leading order QCD. Transverse momentum $\left(p_{\mathrm{T}} \geq 30 \mathrm{GeV}\right)$ and rapidity distributions of the Higgs boson are calculated for the LHC in the large top-quark mass limit. It is pointed out that, as much as in the case of inclusive Higgs production, the $K$-factor of this process is large $(\approx 1.6)$ and depends weakly on the kinematics in a wide range of transverse momentum and rapidity intervals. Our result confirms previous suggestions that the production channel $p+p \rightarrow H+$ jet $\rightarrow \gamma+\gamma+$ jet gives a measurable signal for Higgs production at the LHC in the mass range $100-140 \mathrm{GeV}$, crucial also for the ultimate test of the Minimal Supersymmetric Standard Model.
\end{abstract}

13.85.-t, 14.80.Bm

Typeset using REVTEX

\footnotetext{
*Work partly supported by the EU Fourth Framework Programme 'Training and Mobility of Researchers', Network 'Quantum Chromodynamics and the Deep Structure of Elementary Particles', contract FMRX-CT98-0194 (DG 12 - MIHT) and the Swiss National Foundation.
} 
Recent results from LEP and the SLC indicate that the Higgs boson of the Standard Model might be light. A fit to the precision data has given the values $m_{\mathrm{H}}=76_{-47}^{+85} \mathrm{GeV}$, corresponding to $m_{\mathrm{H}} \leq 262 \mathrm{GeV}$ at the $95 \%$ confidence level, whereas a direct search at LEP200 gives the lower limit as $90 \mathrm{GeV} \leq m_{\mathrm{H}}$ [面]. In addition, a crucial theoretical upper limit exists on the mass of the light neutral scalar Higgs boson of the Minimal Supersymmetric Standard Model $m_{h} \leq 130 \mathrm{GeV}$. It is, therefore, significant that one attempts to get the best possible signals in the light mass range of $100 \mathrm{GeV} \leq m_{\mathrm{H}} \leq 140 \mathrm{GeV}$ at the LHC. Simulation studies carried out by ATLAS and CMS have shown that assuming a low integrated luminosity of $3 \cdot 10^{4} \mathrm{pb}^{-1}$, even in the case of the "gold-plated" decay channel into two photons, the signal significance $(S / \sqrt{B})$ is only around 5 [2]. This conclusion depends on the value of the $K$-factor (a conservative value $K=1.5$ was used) and some plausible assumptions on the size of the background. The calculation of the next-to-leading order (NLO) corrections to the background is not yet complete [3] and the contribution of the NNLO subprocess $g g \rightarrow \gamma \gamma$ is large [4]. The complete NNLO analysis is extremely laborious, but appears to be feasible. Actually, for the full numerical control the background has to be calculated in NNNLO, which is completely beyond the scope of presently available techniques. Fortunately, the ambiguity in the value of the background to the signal is suppressed by the square root appearing in the definition of the signal significance. This situation, which is not completely satisfactory, can be improved by studying the $\gamma+\gamma+$ jet(s) final statest; this offers several advantages. The photons are more energetic than in the case of the inclusive channel and the reconstruction of the jet in the calorimeter allows a more precise determination of the interaction vertex, improving the efficiency and mass resolution. Furthermore the existence of a jet in the final state allows for a new type of event selection and a more efficient background suppression. In addition, the necessary control of the background contributions can probably be already achieved by the inclusion of the NLO corrections (the matrix elements are known [6]). In a recent phenomenological study [7] it has been found that these advantages appear to be able to compensate the loss in production rates, provided one gets a large $K$-factor also for this process. The presentation of the NLO QCD corrections for this process is the main purpose of this letter.

The production process $g g \rightarrow H$ is given by loop diagrams in the Born approximation, since the gluons interact with the Higgs boson via virtual quark loops [8]. The exact calculation of the NLO corrections is rather complex [9]. Fortunately, the effective field theory approach [10 obtained in the large top mass limit with effective gluon-gluon-Higgs coupling gives an accurate approximation (with or without QCD corrections) with an error less than $5 \%$, provided $m_{\mathrm{H}} \leq 2 m_{\mathrm{t}}$ [9,11]. It has been checked in LO, by an explicit calculation, that the approximation remains valid also for the production of Higgs bosons with large transverse momentum, provided both $m_{\mathrm{H}}$ and $p_{\mathrm{T}}$ are smaller than $m_{\mathrm{t}}$ [12]. It is therefore plausible to assume that the approximation remains valid also if we include NLO QCD corrections. Recently, in this approximation and using the helicity method, the transition amplitudes relevant to the NLO corrections have been analytically calculated for all the contributing

\footnotetext{
${ }^{1}$ The study of Higgs production in association with a jet was first suggested in the context of improving $\tau$ reconstruction in the $\tau^{+} \tau^{-}$decay channel [5].
} 
subprocesses (loop corrections [13] and bremsstrahlung [14]).

The available NLO matrix elements contain soft and collinear singularities and therefore do not allow for a direct numerical evaluation of the physical cross section. In the past few years, exploiting the universal structure of the soft and collinear contributions, several efficient algorithms have been suggested to obtain finite cross section expressions from the singular NLO matrix elements. We have used the method of ref. [15] and implemented it into a numerical Monte Carlo style program which allows to calculate any infrared-safe physical quantity for the inclusive production of a Higgs boson with one jet in NLO accuracy.

In this paper we report some of our results obtained for proton-proton collisions with $\sqrt{S}=14 \mathrm{TeV}$. For the strong coupling constant at NLO (LO) we use the standard two-loop (one-loop) form with $\Lambda_{Q C D}$ set to the value used in the analysis of the parton distribution function under consideration. Our default choice for the factorization and renormalization scales is $Q_{0}^{2}=\left(m_{\mathrm{H}}^{2}+p_{\mathrm{T}}^{2}\right)$, where $p_{\mathrm{T}}$ is the transverse momentum of the Higgs boson. Here, unless stated, we consider the case of $m_{\mathrm{H}}=120 \mathrm{GeV}$ and $p_{\mathrm{T}}>30 \mathrm{GeV}$ in the kinematical region where the perturbative result can be applied without having to consider low- $p_{\mathrm{T}}$ resummation effects.

Most of our curves have been obtained with MRST (ft08a) parton distribution functions, but we will also show some results using CTEQ(4M) and GRV98 [16]. To compare the leading with the NLO results, for consistency, we use the corresponding LO parton distributions from each set. We shall discuss only results for the inclusive production cross section of a Higgs boson with large transverse momentum, although as we mentioned above the Monte Carlo program allows to study any infrared-safe quantity, including the implementation of different jet algorithms and experimental cuts.

In Fig. 1(a) we show the $p_{\mathrm{T}}$ distribution of the NLO and LO cross sections using MRST parton densities at three different scales $Q=\mu Q_{0}$, with $\mu=0.5,1,2$. In this figure one can see three important points. First, the radiative corrections are large; second, there is a reduction in the scale dependence when going from LO to NLO; third, the improvement in the scale dependence is still not completely satisfactory. The same features can be observed in more detail in Fig. 1(b), where the LO and NLO cross sections integrated for $p_{\mathrm{T}}$ larger than 30 and $70 \mathrm{GeV}$ are shown as a function of the renormalization/factorization scale. Both the LO and NLO cross sections increase monotonically with decreasing $\mu$ scale, down to the limiting value where perturbative QCD can still be applied. In NLO the scale dependence has a maximum and its position characterizes the stability of the NLO perturbative results. In our case, as a result of the very large positive radiative corrections, the position of the maximum is shifted down to small $\mu$ values where the perturbative treatment is not valid, indicating that the stability of the NLO result is not completely satisfactory. In the usual range of variation of $\mu$ from 0.5 to 2 , however, the LO scale uncertainty amounts up to $\pm 35 \%$, whereas at NLO this is reduced to $\pm 20 \%$, indicating the relevance of the QCD corrections. This feature of the scale dependence in NLO is very much the same as the one in the case of inclusive Higgs production [9]. 

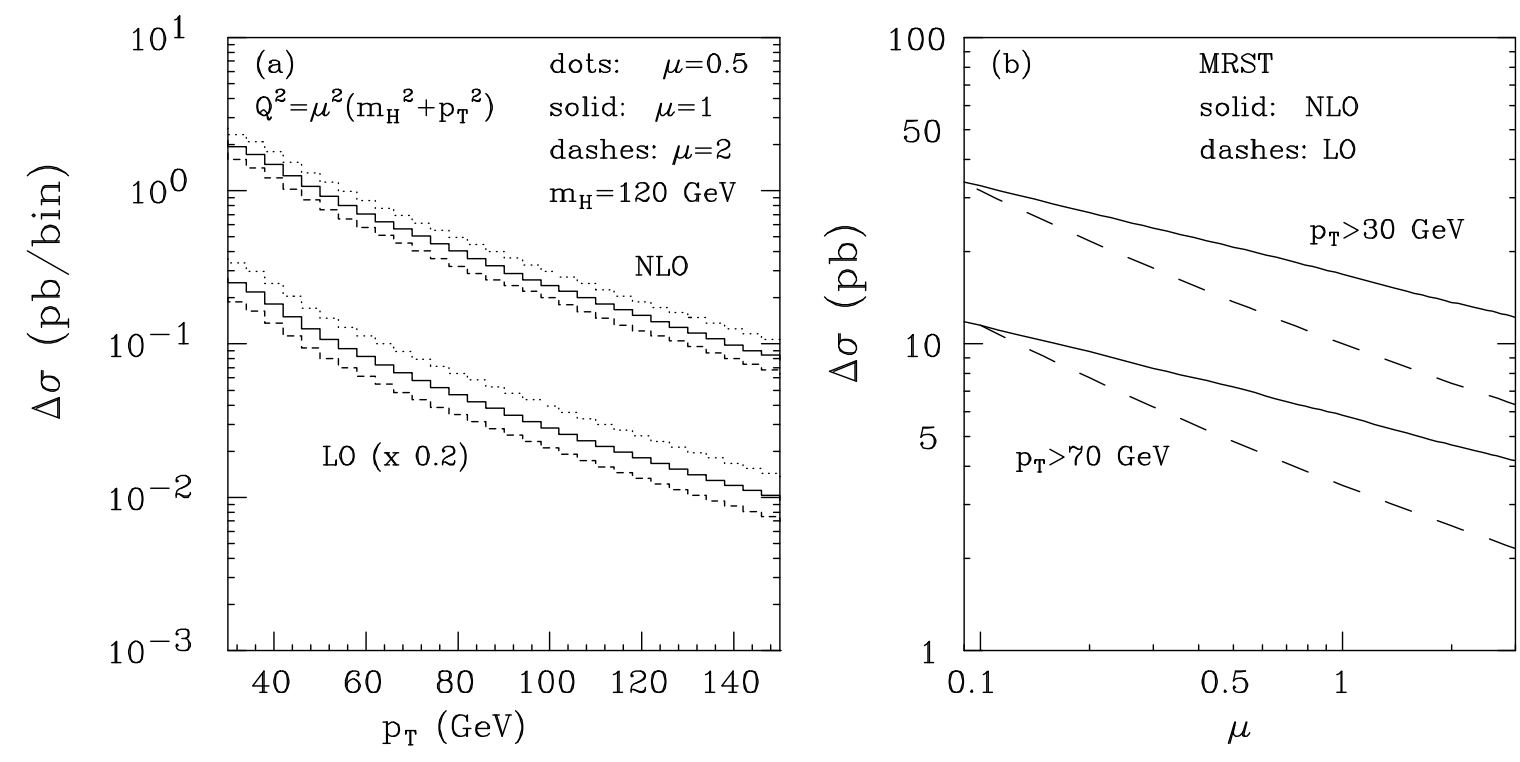

FIG. 1. Scale dependence of NLO and LO distributions using MRST parton densities. (a) $p_{\mathrm{T}}$ distributions at different scales and (b) the scale dependence of the integrated cross sections for $p_{\mathrm{T}}>30$ and $70 \mathrm{GeV}$

In Fig. 2 we show the ratio

$$
K=\frac{\Delta \sigma_{N L O}}{\Delta \sigma_{L O}}
$$

of the next-to leading and the LO cross sections ( $K$-factor) for the three different sets of parton distributions: MRST, CTEQ and GRV98, as a function of the transverse momentum and the rapidity of the Higgs boson. We can see that the $K$-factor is in the range $1.5-1.6$ and it is almost constant (within 15\% accuracy) for a large range of $p_{\mathrm{T}}$ and $y$. In the $p_{\mathrm{T}}$ distribution the variation never exceeds $10 \%$, whereas it is a bit larger for the $y$ distribution at large $|y|$.

The ratios of the NLO cross sections

$$
R=\frac{\Delta \sigma_{\mathrm{CTEQ}, \mathrm{GRV}}}{\Delta \sigma_{\mathrm{MRST}}}
$$

computed by using CTEQ and GRV98 over the one obtained by using MRST parton densities are also shown. From there, it is possible to see that the differences in the $K$-factors basically come from variations in the LO cross sections, mostly because of the value of $\Lambda_{Q C D}$ used in each set.

We conclude that the properties of the $K$-factors found for large transverse momentum Higgs production are very similar to the ones obtained for the total inclusive Higgs production. They are about the same size, they show the same scale dependence, and the $K$-factor changes mildly with changing $m_{\mathrm{H}}, y$ and $p_{\mathrm{T}}$. Its large value and its surprising independence 

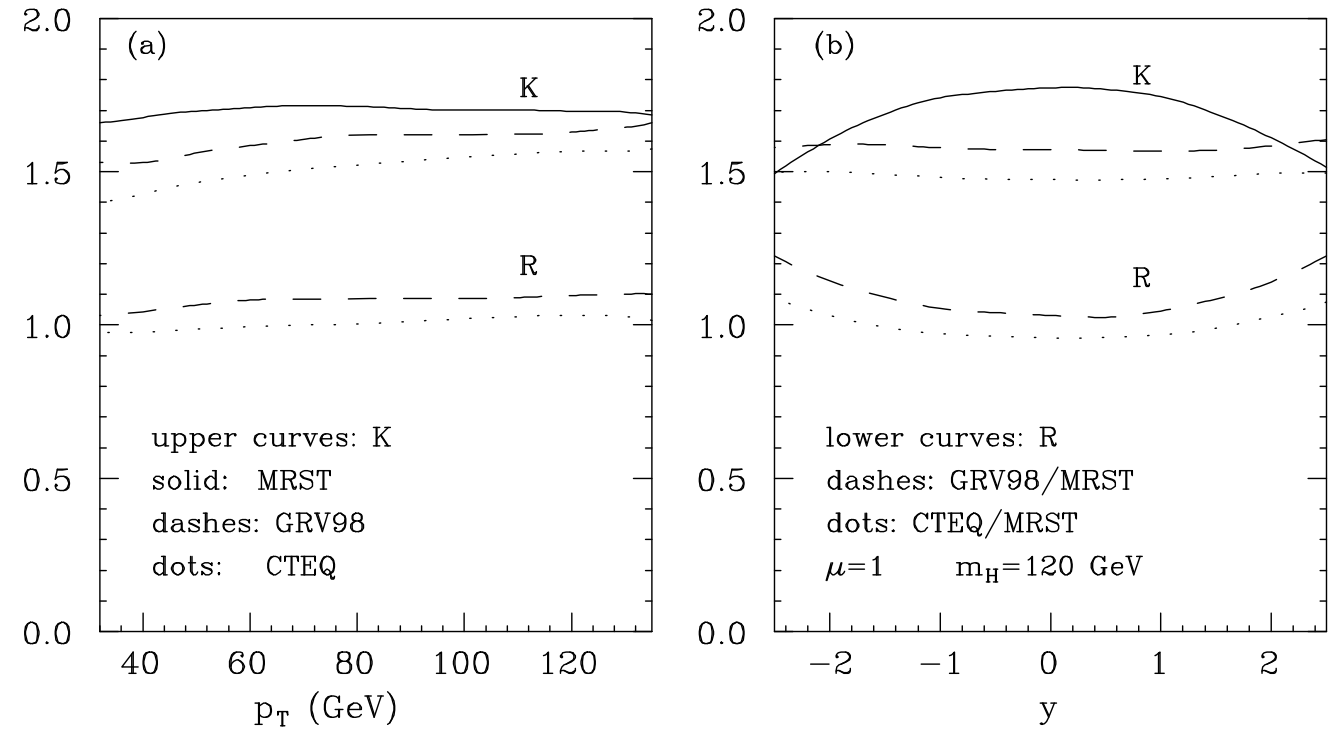

FIG. 2. (a) $p_{\mathrm{T}}$ and (b) $y$ dependence of the $K$-factor and the ratio $R$ of the NLO cross sections for different sets of parton densities (see eqs. (11) and (2)) .

from the kinematics might be interpreted as an evidence for some universal origin of the large radiative corrections [17]. This requires further theoretical understanding.

We have not included in our analysis the contributions from electroweak reactions, which can increase the cross section about $10 \%$ when suitable cuts are applied [7]. Nevertheless it is worth noticing that since QCD corrections to electroweak boson fusion are substantially smaller than the ones corresponding to gluon fusion, the significance of the electroweak contributions is reduced at NLO.

In Fig. 3 we show NLO cross section values for the physics signal $p+p \rightarrow H+$ jet $\rightarrow$ $\gamma+\gamma+$ jet as a function of the Higgs mass (with a reference value for the branching ratio given by $\operatorname{Br}(H \rightarrow \gamma \gamma)=2.18 \cdot 10^{-3}$ for $m_{\mathrm{H}}=120 \mathrm{GeV}$ [18]). For comparison, the cross section values of the physics signal $p+p \rightarrow H \rightarrow \gamma+\gamma$ are also shown. From there it is possible to see that the loss in production rate due to the transverse momentum cut of $p_{\mathrm{T}}>30 \mathrm{GeV}$ is less than a factor of 2 for the range of masses considered.

In conclusion, we have pointed out that, much as in the case of inclusive Higgs production, the cross section values of the associated production of a Higgs boson with a jet are increased by a $K$-factor of $1.5-1.6$ given by NLO QCD radiative corrections. Our result confirms previous suggestions that the production channel $p+p \rightarrow H+$ jet $\rightarrow \gamma+\gamma+$ jet gives a measurable signal for Higgs production at the LHC in the mass range $100-140 \mathrm{GeV}$, crucial also for the ultimate test of the Minimal Supersymmetric Standard Model.

We are grateful to M. Spira for discussions. One of us (DdeF) would like to thank S. Frixione for helpful comments. 


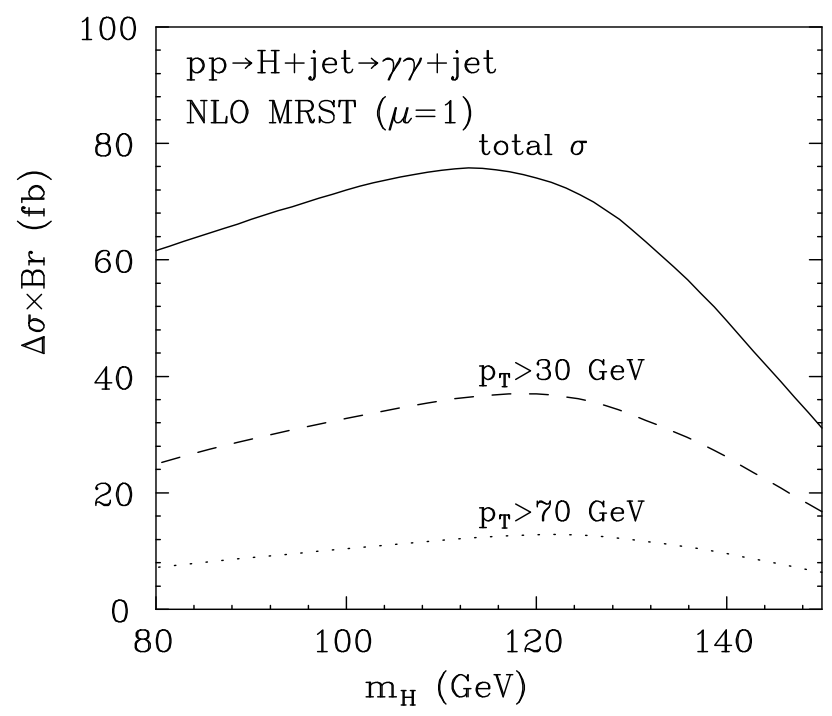

FIG. 3. NLO-integrated cross section for the physics signal $p+p \rightarrow H+$ jet $\rightarrow \gamma+\gamma+$ jet for $p_{\mathrm{T}}>30,70 \mathrm{GeV}$. We also include the value for the total cross section $(p+p \rightarrow H \rightarrow \gamma+\gamma)$.

\section{REFERENCES}

[1] LEP Collaborations and Electroweak Working Group, SLD heavy flavour and electroweak working groups, CERN-EP/99-15.

[2] ATLAS Collaboration, Technical Proposal, CERN/LHCC 94-93 (1994);

CMS Collaboration, Technical Design Report, CERN/LHCC 97-33 (1997) 27;

C. Seez et al., Proc. Large Hadron Collider Workshop, Aachen, CERN-90-10-B/ECFA 90-133, Vol.II, p.474 (1990);

D. Froidevaux, F. Gianotti and E. Richter-Was, ATLAS Internal Note, Phys-No-64 (1995).

[3] P. Aurenche, A. Donini, R. Baier, M. Fontannaz and D. Schiff, Z. Phys. C 29, 459 (1985).

[4] D. Dicus and S. Willenbrock, Phys. Rev. D 37, 1801 (1988);

B. Bailey, J.F. Owens and J. Ohnemus, Phys. Rev. D 46, 2018 (1992).

[5] R.K. Ellis, I. Hinchliffe, M. Soldate and J.J. van der Bij, Nucl. Phys. B 297, 221 (1988).

[6] A. Signer, Phys. Lett. B 357, 204 (1995);

Z. Bern, G. Chalmers, L. Dixon and D. Kosower, Phys. Rev. Lett. 72, 2134 (1994).

[7] S. Abdullin, M. Dubinin, V. Ilyin, D. Kovalenko, V. Savrin and N. Stepanov, Phys. Lett. B 431, 410 (1998).

[8] H. Georgi, S. Glashow, M. Machacek and D. V. Nanopoulos, Phys. Rev. Lett. 40, 692 (1978).

[9] D. Graudenz, M. Spira and P. Zerwas, Phys. Rev. Lett. 70, 1372 (1993);

M. Spira, A. Djouadi, D. Graudenz and P. Zerwas, Nucl. Phys. B 453, 17 (1995).

[10] S. Dawson, Nucl. Phys. B 359, 283 (1991);

A. Djouadi, M. Spira and P. Zerwas, Phys. Lett. B 264, 440 (1991).

[11] S. Moretti, Z. Kunszt and W.J.Stirling, Z. Phys. C 74479 (1997).

[12] U. Baur and E. Glover, Nucl. Phys. B 339, 38 (1990). 
[13] C. Schmidt, Phys. Lett. B 413, 391 (1997).

[14] S. Dawson and R. Kauffman, Phys. Rev. Lett. 68, 2273 (1992);

R. Kauffman, S. Desai and D. Risal, Phys. Rev. D 55, 4005 (1997).

[15] S. Frixione, Z. Kunszt and A. Signer, Nucl. Phys. B 467, 399 (1996);

S. Frixione, Nucl. Phys. B 507, 295 (1997).

[16] A.D. Martin, R.G. Roberts, W.J. Stirling and R.S. Thorne, Eur. Phys. J C4, 463 (1998);

M. Glück, E. Reya and A. Vogt, Eur. Phys. J C5, 461 (1998);

H. Lai et al., Phys. Rev. D 55, 1280 (1997).

[17] M. Krämer, E. Laenen and M. Spira, Nucl. Phys. B 511, 523 (1998).

[18] A. Djouadi, J. Kalinowski and M. Spira, Comp. Phys. Commun. 108, 56 (1998). 